\title{
Collective minds
}

\section{By tapping into social cues, individuals in a group may gain access to higher-order computational capacities that mirror the group's responses to its environment.}

\section{Iain Couzin}

In 1905 the field naturalist Edmund Selous, a confirmed Darwinian and meticulous observer of bird behaviour, wrote of his wonderment when observing tens of thousands of starlings coming together to roost: "they circle; now dense like a polished roof, now disseminated like the meshes of some vast all-heaven-sweeping net...wheeling, rending, darting... a madness in the sky".

Throughout his life Selous struggled to explain the remarkable synchrony and coherence of motion during flocking, and he concluded that somehow a connectivity of individual minds and transference of thoughts must underlie such behaviour. "They must think collectively, all at the same time, or at least in streaks or patches - a square yard or so of an idea, a flash out of so many brains".

We now know that such synchronized group behaviour is mediated through sensory modalities such as vision, sound, pressure and odour detection. Individuals tend to maintain a personal space by avoiding those too close to themselves; group cohesion results from a longer-range attraction to others; and animals often align their direction of travel with that of nearby neighbours. These responses can account for many of the group structures we see in nature, including insect swarms and the dramatic vortex-like mills formed by some species of fish and bat. By adjusting their motion in response to that of near neighbours, individuals in groups both generate, and are influenced by, their social context - there is no centralized controller.

But when observing a starling flock, or a sweeping, twisting school of tiny silvered fish, I often think of Selous's concept of a collective mind. It is perhaps too easy to disregard his vision, based as it was on a Victorian fascination with telepathy. Indeed, his rich descriptions capture the essence of something more, something we still know very little about: how social interactions affect the way animals within highly coordinated groups acquire and process information.

For individuals within groups, survival can depend critically on how local behavioural rules scale to collective properties. Pertinent information, such as the location of resources or predators, may often be detected by only a relatively small proportion of group members due to limitations in individual sensory capabilities, often further restricted by crowding. Close behavioural coupling among near neighbours, however, allows a localized change in direction to be amplified, creating a rapidly growing and propagating wave of turning across the group. This positive feedback results from the ability of indi-

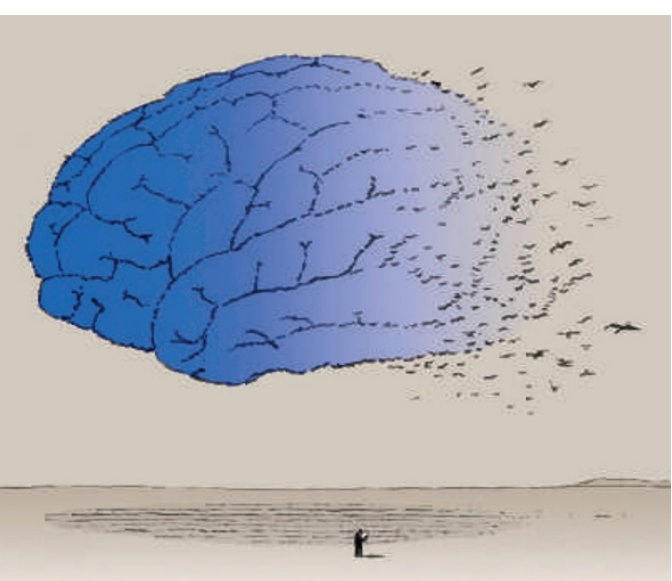

viduals to influence and be influenced by others, and allows them to experience an 'effective range' of perception much larger than their actual sensory range.

The scaling from actual to effective sensory range is non-linear, however. It is hard for groups to remain cohesive and for information to spread if individuals respond only to others very close to themselves. As sensory range is increased, a response to a greater number of neighbours increases cohesion and allows effective long-range transfer of directional information. If this range expands further still, groups that form are highly cohesive but individuals may get misdirected, as the motion of distant individuals is less likely to encode relevant information about localized stimuli.

Individuals within groups may modify their interactions in a context-dependent way. Under threat of attack, for example, individuals often align more strongly with one another, heightening collective sensitivity to weak or ambiguous environmental stimuli, and so increasing the 'system gain. However, amplification can occur in response to random fluctuations, creating false alarms that can be costly.

Under different circumstances individuals may adopt behaviour that facilitates collective damping of local fluctuations. During long-distance migration, for example, animals are often faced with the challenge of navigating up noisy and weak thermal or resource gradients. Local variability makes this task difficult, or even impossible, for individuals in isolation. But coherent social interactions can allow groups to function like an integrated self-organizing array of sensors, again increasing effective perceptual range. As long as interactions are sufficiently sensitive to ensure cohesion, but not too sensitive to local fluctuations and individual error, individuals can effectively respond to the weak long-range gradient.

We are beginning to comprehend more fully how individuals in groups can gain access to higher-order collective computational capabilities such as the simultaneous acquisition and processing of information from widely distributed sources. Group members may come to a consensus not only about where to travel but also about what local rules to use. Thus, like the brain, groups may adapt to compute 'the right thing' in different contexts, matching their collective information-strategy with the statistical properties of their environment.

Selous wrote in despair of his contemporaries' lack of interest in flocking: "If there really were anything extraordinary in the collective movements of birds...they would have been much discussed and much wondered at". But today there is a rapidly expanding and vibrant community of biologists, engineers, mathematicians and physicists for whom flocking serves as inspiration. Such group behaviour holds clues about the evolution of sociality, and also for the development of novel technological solutions, from autonomous swarms of exploratory robots to flocks of communicating software agents that help each other to navigate through complex and unpredictable data environments. lain Couzin is in the Department of Zoology, University of Oxford, Oxford OX13PS, UK.

FURTHER READING

Conradt, L. \& Roper, T. J. Trends Ecol. Evol. 20, 449-456 (2005).

Couzin, I. D. \& Krause, J. Adv. Study Behav. 32, 1-75 (2003). Couzin, I. D., Krause, J., Franks, N. R. \& Levin, S. A. Nature 433, 513-516 (2005).

Sumpter, D. J. T. Phil. Trans. R. Soc. B 361, 5-22 (2006).

For other essays in this series, see http:// nature.com/nature/focus/arts/connections/ index.html 